\title{
THz Instruments for Space
}

\author{
Peter H. Siegel, Fellow, IEEE
}

\begin{abstract}
Terahertz technology has been driven largely by applications in astronomy and space science. For more than three decades cosmochemists, molecular spectroscopists, astrophysicists, and Earth and planetary scientists have used submillimeter-wave or terahertz sensors to identify, catalog and map lightweight gases, atoms and molecules in Earth and planetary atmospheres, in regions of interstellar dust and star formation, and in new and old galaxies, back to the earliest days of the universe, from both ground based and more recently, orbital platforms. The past ten years have witnessed the launch and successful deployment of three satellite instruments with spectral line heterodyne receivers above $300 \mathrm{GHz}$ (SWAS, Odin, and MIRO) and a fourth platform, Aura MLS, that reaches to $2520 \mathrm{GHz}$, crossing the terahertz threshold from the microwave side for the first time. The former Soviet Union launched the first bolometric detectors for the submillimeter way back in 1974 and operated the first space based submillimeter wave telescope on the Salyut 6 station for four months in 1978. In addition, continuum, Fourier transform and spectrophotometer instruments on IRAS, ISO, COBE, the recent Spitzer Space Telescope and Japan's Akari satellite have all encroached into the submillimeter from the infrared using direct detection bolometers or photoconductors. At least two more major satellites carrying submillimeter wave instruments are nearing completion, Herschel and Planck, and many more are on the drawing boards in international and national space organizations such as NASA, ESA, DLR, CNES, and JAXA. This paper reviews some of the programs that have been proposed, completed and are still envisioned for space applications in the submillimeter and terahertz spectral range.
\end{abstract}

Index Terms-Space instruments, submillimeter wave, terahertz (THz).

\section{INTRODUCTION}

W HILE MINDFUL of the flurry of recent activities in fields as diverse as security screening, ultra fast chemistry, and biological imaging, terahertz technology has been driven largely by applications in astronomy, Earth and planetary science. Extreme atmospheric opacity from pressure broadened water and oxygen vibrational and rotational absorption signatures prevents terahertz energy from being propagated great distances under standard temperature and pressure. Even observing relatively strong naturally occurring terahertz emission signatures from nearby star forming regions or planets requires going above most of the atmosphere, either to high and dry mountain top observatories, stratospheric aircraft and balloons, or orbital platforms. However, once the attenuation has been reduced, the wealth of information about our own atmospheric processes, the constituents and conditions in the atmospheres of solar system bodies, and the vast amount of cosmic chemistry that occurs both around and within stellar

Manuscript received October 2, 2006

The author is with the Division of Biology, Beckman Institute and the NASA Jet Propulsion Laboratory, California Institute of Technology, Pasadena, CA 91125 USA (e-mail: phs@ caltech.edu).

Digital Object Identifier 10.1109/TAP.2007.908557 systems, all provide a strong motivating force for the development and deployment of terahertz sensors. As a consequence, a considerable amount of scientific resources have been invested in terahertz instruments, including three deployed space heterodyne instruments collecting high resolution spectra up to $600 \mathrm{GHz}$ : the Submillimeter Wave Astronomy Satellite (SWAS), NASA (490/550 GHz); Odin, Sweden (490/580 GHz) and Microwave Instrument for the Rosetta Orbiter (MIRO), ESA (190/560 GHz); and a fourth Earth sounding instrument, Microwave Limb Sounder (MLS) on NASA's Aura satellite, with capabilities at $118,190,240,640$, and $2520 \mathrm{GHz}$. There are also four broadband far-infrared instruments that have been launched in the west and one in Japan carrying cooled spectrophotometers and bolometric detectors: Infrared Astronomical Satellite (IRAS) [40-100 microns], NASA; Diffuse IR Background Experiment (DIRBE) [1-240 microns], and Far Infrared Absolute Spectrophotometer (FIRAS) [30 GHz to $2.9 \mathrm{THz}]$, all on the Cosmic Background Experiment (COBE), NASA; Infrared Space Observatory (ISO), [50-200 microns], ESA; and Multiband Imaging Photometer (MIPS) [50-160 microns] on the Spitzer Space Telescope, NASA; and most recently Akari (formerly ASTRO-F) just launched in February 2006 by JAXA (Japan) which goes out to 180 microns. At least two other submillimeter wave spectrophotometers were flown on platforms developed in the former Soviet Union-one on Kosmos 669 in 1974 and a full submillimeter wave telescope system (BST-1 M) with detectors in the range from 60-1000 microns. BST-1 $\mathrm{M}$ was operated for several months on the Salyut 6 space station in 1978. These instruments provided a first look at the far-infrared universe. Many more Earth, planetary and space science instrument proposals are due to be decided upon over the next one to five years, and plans for more grandiose missions dot the pages of both NASA and ESA study packages. This paper reviews some of the past, present and future applications and missions for terahertz space science instruments. Both successful and ultimately unfunded proposals are referenced. Future opportunities are highlighted and it is for the reader to judge whether these will have a chance to be realized within the backdrop of continuing (or diminishing) public support for everything space.

\section{UniQue SPACE APPLICATIONS FOR THz SCIENCE}

Molecular line spectroscopy, the detection, identification and mapping of thermal emission and absorption signatures from lightweight low pressure gases, forms the basis for much of the science that has motivated the development of terahertz space instruments [1]. In the cool regions around star systems, in the atmospheres of planets, within the large deposits of gas and dust that lie along the spiral arms and in the central regions of galaxies, thermally excited atoms and molecules radiate (or absorb) strong narrow band signals corresponding 
to quantum state transitions associated with specific rotational and vibrational modes. In addition, for temperatures roughly in the 10-100 $\mathrm{K}$ range, continuum blackbody energy from thermally illuminated gas and dust has its peak output in the terahertz frequency range. Finally, optical energy from very distant galaxies is red shifted down into the terahertz bands, where when detected, serves as a probe into the state and composition of the early universe. The measurement of individual or ratio'd pairs of spectral emission or absorption lines from various planetary, stellar or galactic sources, provides data on the origin, composition, evolution, environment and chemical reactions that are responsible for the universe as we know it. Excellent overviews of the basic science motivations for terahertz sensor development can be found in several of the large astrophysics mission proposals that have been developed since the mid 1990s, many of which are summarized in the online proceedings of an infrared space astrophysics conference held recently in Pasadena, CA, [2].

Looking back towards the Earth, terahertz measurements help to generate and validate models for understanding dynamic processes in our own atmosphere, such as those involved in ozone depletion, distribution of both natural and man-made pollutants, radiation balance and global warming through water vapor and temperature profiling [3].

Similar data can be gleaned from the atmospheres of planets, moons, comets and asteroids, including the search for chemical signatures associated with subsurface volcanic activity or even life processes [4], [92]. Temperature, pressure, and wind velocity in addition to chemical mixing ratios are all retrievable parameters using narrow band heterodyne radiometry [5].

Measuring the exact spectral line peak wavelength, profiling the shape and any fine structure, and calibrating the strength against a known background or target, provide species identification, surrounding pressure, temperature and velocity information, as well as the presence or absence of magnetic fields. Such a wide variety of information can be obtained from these measurements and over such a wide range of objects-from distant galaxies to our own planet-that there is a strong push for instruments that can perform these observations without the intervening absorption and atmosphere induced turbulence that affects ground based instruments. Already more than half a dozen orbital platforms have been designed and launched with capabilities to record these spectral signatures. This is on top of a very substantial worldwide investment in mountain top radio telescope facilities, high altitude balloon and aircraft platforms and even telescopes manned year round at the South Pole. For this paper we confine ourselves to space facilities only.

\section{Historic AND OPERATIONAL THz SPACE MisSiONS}

Microwave observations from space date back to the earliest days of flight, when Mariner 2 used radiometers at $15.8 \mathrm{GHz}$ (surface temperature) and $22.2 \mathrm{GHz}$ (water vapor) to examine Venus in a 1962 flyby. Millimeter-wave observations (>30 GHz) of the Earth from space to look at global rainfall, cloud cover, atmospheric temperature and pressure and water vapor distribution have been ongoing since the Soviet Cosmos series of the late 1960s (Cosmos 243-1968 and 384-1970) and the U.S. Nimbus satellites (starting with Nimbus 5) and
Tyros platforms which spanned the 1970s. An excellent summary of these early missions can be found in [6]. Even during this time period the strong water absorption line at $183 \mathrm{GHz}$ was much appreciated [7] although it was not mapped from satellites (at least in the west) until the early 1990s when NASA's Upper Atmospheric Research Satellite Microwave Limb Sounder (UARS-MLS) instrument [8], [9] and the U.S. Defense Meteorological Satellite Program's (DMSP) Special Sensor Microwave Water Vapor Profiler (SSM/T-2) sounder [10], [11] were launched and successfully deployed.

Soviet submillimeter-wave space observations began in the early 1970's when a strong development team at the Lebedev Institute of Physics and Astronomy realized the importance of terahertz spectroscopy for examining star forming regions as well as for understanding galactic structure and evolution [12]. They also appreciated the need to get above the Earth's atmosphere in order to make sensitive observations. As a result, the very first space-based submillimeter wave radiometer system was flown on board the Kosmos 669 satellite [13], [14] and was used to make the first far infrared observations of the radiant thermal energy from the Earth. This significant technological feat was followed shortly after by a full blown submillimeter wave telescope flown and operated on the Salyut 6 space station between June and September of 1978 . The instrument had a $1.56 \mathrm{~m}$ optical Cassegrain telescope and a 3 stage Joule Thomson $4.2 \mathrm{~K}$ active cooler housing germanium and indium antimonide bolometers operating between 60-130 and 300-1000 microns respectively with sensitivities of $10^{-12} \mathrm{~W} / \sqrt{ } \mathrm{Hz}$ [14].

United States interest in submillimeter-wave space science dates back at least to the days in the early 1970s [15]-[17] when the first large diameter millimeter-wave ground based radio telescopes were turned to the gaseous star forming regions in the Orion nebula [18] and the atmosphere of Venus [19]. Breakthroughs both in cooled detector technology and spectroscopic techniques enabled a diverse suite of both ground based and high altitude balloon and aircraft measurements [20]. Very strong and omnipresent molecular spin transition lines of carbon monoxide, $\mathrm{J}=\mathrm{n}$ to $\mathrm{n}-1, \mathrm{n}=1$ to 7 , at 115,230 , $345 \mathrm{GHz}$, etc., up to at least $690 \mathrm{GHz}$ [21] became primary targets for these early observations. These and higher frequency transitions were also measured from high altitude aircraft such as the Kuiper Airborne Observatory [22] and stratospheric balloon platforms [23]. The galactic spiral arms as well as many new star forming regions were also mapped at these wavelengths [16]. At the same time hundreds of additional spectral signatures, many from atomic and molecular species not stably reproducible on Earth, were recorded and compared to laboratory data to identify those unique and common forms that exist in the low pressure low temperature environment of space [24].

The first terahertz space platforms focused not on discrete, fully resolvable spectral lines, but on continuum observations or broad spectral surveys using cooled direct detection bolometers, Fourier transform spectrometers and spectrophotometers. This was due both to the need for general survey information before specific molecules could be targeted, and to the lack of available spectral line receiver technology, which was not well developed in the submillimeter until the mid 1990's. The first infrared 
telescope devoted to space science launched in the west [a collaboration between NASA (U.S.), NIVR (The Netherlands) and SERC (U.K.)] was the Infra-Red Astronomy Satellite (IRAS) which carried a helium cryogen that lasted for approximately nine months (late January to late November, 1983) and allowed the spacecraft to make observations in four bands between 8 and 120 microns over $98 \%$ of the sky [25]. IRAS carried a Cassegrain (Ritchey-Chretien) telescope with a $57 \mathrm{~cm}$ diameter beryllium mirror cooled to $10 \mathrm{~K}$ to reduce background noise. It employed small arrays of silicon and germanium based bolometers cooled to below $3 \mathrm{~K}$. This first extensive survey of the infrared universe produced many exciting discoveries, one of the most relevant to this community being that in at least some spiral galaxies, $50 \%$ of the luminosity and $98 \%$ of the photons are being radiated in the far IR [26].

Following in the very successful footsteps of IRAS, the European Space Agency (with collaboration from NASA and ISAS the Institute of Space and Astronautical Science of Japan) developed and launched the next generation infrared observatory known as the Infrared Space Observatory (ISO) in November 1995 [27], [28]. ISO carried 2300 liters of super fluid helium that kept a $60 \mathrm{~cm}$ Ritchey-Chretien telescope at between 2-8 K during a 28 month long mission to observe between 2 and 240 microns with a suite of four instruments. The far infrared was covered by a $2-240$ micron photometer with resolution $(f / \Delta f)$ between 50 and 30000 and a long wavelength spectrometer operating from 43 to 200 microns with resolution between 150 and 9700. The spectrometer used a Fabry Perot for frequency resolution and 1.8-3 $\mathrm{K}$ beryllium and gallium-doped germanium photoconductors for detection [29]. Among ISO's many discoveries and accomplishments were the first measurements of HF in interstellar space, the detection of water in galaxies as well as star forming regions, the detection of $\mathrm{HD}$ and measurements of the deuterium to hydrogen ratio $(\mathrm{D} / \mathrm{H})$ in planetary atmospheres, and many detailed spectral surveys of galaxies, star forming regions and solar system bodies [30].

In between IRAS and ISO, NASA launched a third infrared satellite, Cosmic Background Explorer (COBE) in November 1989 targeted at measurements of the early universe. COBE carried three major instruments, a Far Infrared Absolute Spectrophotometer (FIRAS) to compare the cosmic microwave background (CMB) against a precise blackbody, Differential Microwave Radiometer (DMR) to map the CMB, and Diffuse Infrared Background Experiment (DIRBE) to search for the cosmic infrared background (CIB) [31]. DMR is a microwave instrument $(30-50 \mathrm{GHz})$. FIRAS and DIRBE use composite bolometers at $1.5 \mathrm{~K}$ for their long wavelength channels which span to 300 microns in the case of DIRBE, a filtered multiband photometer, and all the way from 100 microns $(3 \mathrm{THz})$ to $10 \mathrm{~mm}(30 \mathrm{GHz})$ for the polarizing Michelson interferometer on FIRAS. COBE's operational mission ran approximately four years and from the very start it delivered spectacular findings about the cosmic background and the development of the early universe. The predicted blackbody spectrum of the $\mathrm{CMB}$ resulting from the Big Bang model were confirmed early on in the mission and the anisotropy of the CMB was also clearly observed. FIRAS made millions of detailed spectral observations across the sky detecting many atomic and molecular species for the first time. More than ten years later new results are still being mined from the data and the reader might periodically check various COBE websites for the most up to date information, e.g., [32]. COBE was followed by the Wilkinson Microwave Anisotropy Probe (WMAP) launched in 2001 [33] which continued even more detailed mapping of the early universe but using microwave observations up to $90 \mathrm{GHz}$.

The latest NASA instrument in the far-infrared astronomical mission queue is the Spitzer Space Telescope (formerly the Space Infrared Telescope Facility-SIRTF) which was launched by NASA in August 2003 [34], [35]. Under development since the early 1980s, Spitzer houses an $85 \mathrm{~cm}$ diameter cooled beryllium Ritchey-Chretien telescope and three cryogenically-cooled (super fluid helium) science instruments, two of which operate in the mid-to-far infrared. An IR spectrograph covers 5 to 40 microns in four low and high resolution bands and a multiband photometer uses arrays centered at $24,70,160$, and 50-100 microns. Amongst its many scientific achievements, Spitzer has made the first images of a brown dwarf and planet orbiting a sun-like star, and detected the chemical building blocks of proteins-hydrogen cyanide, acetylene and $\mathrm{CO}_{2}$ [36], the first two of which, it might be noted, when combined with water and an appropriate reaction surface, also present in interstellar dust, can form the DNA purine base adenine [37].

The latest infrared instrument to reach orbit is Japan's Akari (formerly ASTRO-F) which carries a $68 \mathrm{~cm}$ cooled telescope $(6 \mathrm{~K})$ and Ge:Ga far IR photometers and imaging spectrometers operating through a Fourier Transform spectrometer out to 180 microns [38]. Akari was launched in February 2006 and is currently operational, with a goal of performing an all sky infrared survey (like IRAS).

As previously implied, $\mathrm{THz}$ instruments using heterodyne sensors for high resolution spectroscopy $\left(f / \Delta f>10^{6}\right)$ lagged behind technologically the broader bandwidth bolometeric and photometric detectors. However these instruments began to reach orbital platforms in the late 1990s with the first such submillimeter wave system, Submillimeter Wave Astronomical Satellite (SWAS) launched by NASA in December 1998 [39] under the Small Explorer program. SWAS carries a $55 \times 71 \mathrm{~cm}$ elliptical off-axis Cassegrain telescope with two radiatively cooled $(170 \mathrm{~K})$ Schottky diode receivers at 490 and $550 \mathrm{GHz}$ for the detection of primarily water $\left(\mathrm{H}_{2} \mathrm{O}\right.$ at $557 \mathrm{GHz}$ and $\mathrm{H}_{2}^{18} \mathrm{O}$ at $\left.547 \mathrm{GHz}\right)$, oxygen $\left(\mathrm{O}_{2}\right.$ at $\left.487 \mathrm{GHz}\right)$, carbon $(\mathrm{CI}$ at $492 \mathrm{GHz})$ and carbon monoxide $\left({ }^{13} \mathrm{CO}\right.$ at $\left.551 \mathrm{GHz}\right)$. Although turned off in 2004, it was rekindled for observations of NASA's Deep Impact probe as it crashed into the nucleus of comet 9P/Tempel-1 in 2005 [40].

A second submillimeter wave heterodyne instrument, Odin (Swedish Space Corporation [41]), was assembled and launched by teams from Sweden, Canada, France, and Finland in February 2001 [42]. This small but technically very ambitious satellite covers $486-504 \mathrm{GHz}$ and $541-580 \mathrm{GHz}$ with four cooled Schottky diode heterodyne radiometers. An additional heterodyne channel at $118 \mathrm{GHz}$ (for oxygen) has been implemented with a cooled MMIC InP HEMT pre-amplifier. The receiver operating temperature of about $120 \mathrm{~K}$ is realized by a closed cycle Sterling cooler and the backend spectrometer includes two hybrid autocorrelators and an acousto-optic 
spectrometer. Observation modes include both limb scanning via satellite nodding (Earth atmosphere) and space viewing, and cover a large cadre of important aeronomy and astronomy spectroscopic lines. After more than six years of operation (still continuing at time of writing despite a variety of operational problems [43]) Odin has monitored chlorine and ozone in the stratosphere longer than any other satellite, has observed more than 70 new spectral lines, looked at emission from more than a dozen comets and measured water in the upper atmosphere of Mars [44], [45].

The first space-based heterodyne observations at $\mathrm{THz}$ frequencies are being made on NASA's Aura satellite by the Microwave Limb Sounder (MLS) instrument [46]. MLS went operational in July 2004 and fields five spectral line radiometers between 118 and $2520 \mathrm{GHz}$. The middle channels (190, 240, and $640 \mathrm{GHz}$ ) employ room temperature Schottky diode downconverters and Gunn diode pumped multiplier chains for the local oscillators. The $118 \mathrm{GHz}$ channel uses an InP HEMT pre-amplifier and downconverter. The $\mathrm{THz}$ radiometer, centered at $2520 \mathrm{GHz}$ with a 6-23 GHz intermediate frequency, uses a planar Schottky diode waveguide mixer (mixer noise $<5000 \mathrm{~K}$ DSB) but is driven by a fully autonomous $\mathrm{CO}_{2}$ pumped far-IR methanol gas laser that outputs more than $20 \mathrm{~mW}$ at $2522 \mathrm{GHz}$ [47]. Aura MLS is targeted at stratospheric chemistry and is globally mapping and monitoring dozens of key chemical species involved in global warming, ozone depletion, solar radiation balance (through ice crystal detection) and distribution of pollutants and aerosols.

A fourth submillimeter-wave heterodyne instrument, MIRO, the first devoted to solar system objects, was launched in March 2004 as part of ESA's Rosetta mission. The spacecraft will rendezvous and land a probe on Comet 67P Churyumov-Gerasimenko in November 2014. MIRO [48] carries 562 spectral line and $188 \mathrm{GHz}$ continuum radiometers for measurements of water, carbon monoxide, ammonia and methanol in the comet tail as well as the coma and nucleus [49]. The submillimeter wave receiver technology is similar to that used on Aura MLS at $640 \mathrm{GHz}$.

At least one more space-bound submillimeter wave heterodyne instrument has been completed but has not yet flown. It is worth mentioning because it was to be the first to utilize high sensitivity receiver technology based on niobium superconductor insulator superconductor tunnel junctions [50]. The instrument was developed and built by NASDA (now JAXA), the Japan Space Agency, for the Japanese Experiment Module (JEM) on the International Space Station. Completed in 2005 the SMILES (Superconducting Submillimeter Wave Limb Emission Sounder) has a pair of superconducting radiometers spanning 625-650 GHz and a mechanical two stage Stirling cooler with a $4 \mathrm{~K}$ Joule Thomson head. The targeted application was stratospheric chemistry (matching the MLS $640 \mathrm{GHz}$ frequency band) and launch had been set for 2008 [51]. However the complete instrument is currently sitting in storage waiting for a final deployment decision on the International Space Station (ISS).

Based on their very early work with submillimeter wave instruments, other missions may have been developed or even fielded in the former Soviet Union, but the author has no additional information on these. The current generation of $\mathrm{THz}$ space instruments-being assembled as this paper is being written-is spearheaded by the European Space Agency (ESA) as the Fourth Cornerstone Mission in their Horizon 2000 program: the Herschel Space Observatory. It is by far the most ambitious of the far infrared missions to date.

\section{SPACE THz Missions IN PREPARATION}

The European Space Agency's Far Infrared and Submillimeter Telescope (FIRST), now the Herschel Space Observatory, and a competing NASA mission, Large Deployable Reflector (LDR), were both given the go-ahead in the mid 1980s (although the LDR concept formally dates back to a JPL study from 1977 [52]). LDR, as the name implies, targeted the far infrared region with a deployable telescope of unprecedented aperture- $20 \mathrm{~m}$, in order to achieve resolving powers sufficient for protoplanets and protogalaxies [53], [54]. FIRST [55] was somewhat less ambitious on the aperture realization-8 $\mathrm{m}$, but was to use an unfolding segmented Cassegrain with room temperature and cryogenic heterodyne detectors from $1 \mathrm{~mm}$ to 100 microns. FIRST was part of Europe's Horizon 2000 program (later becoming the fourth Cornerstone Mission [56] in this suite, along with Cluster and Solar Heliospheric Observatory (SOHO), Newton, and Rosetta) and received substantial funding in 1986. Following a strong National Academy of Sciences recommendation in 1982, NASA finally gave a go ahead for LDR in 1987 along with a companion suite of smaller precursor missions that included SWAS and indirectly the Stratospheric Observatory for Infrared Astronomy (SOFIA) - the high altitude aircraft platform that is to replace the NASA Kuiper Airborne Observatory. Other MIDEX and SMEX (Middle and Small Explorer programs) instruments were also placed in the NASA mission queue for a short time but none have yet materialized as funded programs. LDR had been planned as a follow on to the Space Infrared Telescope Facility (SIRTF) [now, the Spitzer Space Telescope], however, as the acronym implied, the European FIRST mission (Herschel) will launch first, in 2008 if everything continues as planned. The closest current NASA equivalent of LDR [Single Aperture Far-Infrared Observatory (SAFIR)] will have to wait at least another decade.

The Herschel Space Observatory [57] specifically targets the far infrared and has both direct detection imaging bolometers and photometers as well as seven high resolution heterodyne spectrometer bands covering 480 to $1910 \mathrm{GHz}$. The original $8 \mathrm{~m}$ ambient temperature deployable aperture slated for FIRST has been replaced with a much lighter $3.5 \mathrm{~m}$ diameter solid dish aluminum coated silicon carbide telescope with better than 10 micron surface accuracy and cooled to $80 \mathrm{~K}$ to lower the background noise for the bolometers [58]. The submillimeter wave heterodyne instrument package-Heterodyne Instrument for the Far Infrared (HIFI) [59]-[61] is both extremely complex (seven separate frequency diplexed spectral channels) and technically ambitious (all $4 \mathrm{~K}$ operation) with superconducting insulator superconducting (SIS) tunnel junction detectors between 480 and $1250 \mathrm{GHz}$ and hot electron bolometer (HEB) mixers for two channels between 1.4 and 1.9 THz. Not only are both of these detector technologies being deployed in space for the 
first time, most of the solid-state local oscillator sources (power amplifier and Schottky diode multiplier chains operating at 120 $\mathrm{K}$ ) and several of the SIS and HEB mixers are being developed solely for this mission and have not been deployed anywhere before. Challenges at the component as well as the subsystem abound but are being quickly overcome as the launch window (now 2008) rapidly approaches. HIFI has been a worldwide development effort with participants from many laboratories in Europe as well as the U.S. At this time all the receiver bands have been delivered to the HIFI project headquarters at SRON (Netherlands Institute for Space Research), where integration and test has been ongoing for over a year. The heterodyne detector noise performance is targeted at $3 \mathrm{X}$ the quantum limit $(\mathrm{h} v / \mathrm{k})$ for all channels up to $1250 \mathrm{GHz}$. Key molecular lines include NII at 1.4 and CII near $1.9 \mathrm{THz}$ but the instrument is capable of tuning continuously and with a resolution $(f / \Delta f)$ of greater than $10^{7}$. The telescope will orbit at the Lagrange 2 point beyond the Moon and is expected to operate for $>36$ months.

Two additional infrared instruments, Spectral and Photometric Imaging Receiver (SPIRE) and Photodetector Array Camera and Spectrometer (PACS) will be carried on Herschel. SPIRE [62] uses a unique array of photolithographed spiderweb bolometers [63] made of neutron transmutation doped (NTD) germanium on a silicon nitride mesh and cooled to 0.3 $\mathrm{K}$ for imaging between 200 and 500 microns through a tunable Mach-Zender interferometer. PACS [64] employs cooled germanium-gallium photoconductor and silicon bolometer arrays to perform imaging and spectroscopy from 60-200 microns.

Herschel has pushed the state-of-the-art in both receiver and source technology in the submillimeter and represents an outstanding achievement in infrared technology. It will launch with a second major instrument Planck ${ }^{1}$ [65], [66], that will observe in the millimeter and submillimeter regions as a follow-on to COBE and WMAP, targeting cosmic background physics. Planck has a $1.5 \mathrm{~m}$ off-axis paraboloidal reflector system with two instrument packages, Low Frequency Instrument (LFI) with cooled $20 \mathrm{~K}$ HEMT amplifiers at 33,44 , and $70 \mathrm{GHz}$ and a high frequency instrument (HFI) with $0.1 \mathrm{~K}$ bolometer arrays between 100 and $857 \mathrm{GHz}$.

The submillimeter-wave frequency regime is also being targeted by major new ground based and aircraft astronomical observatory programs notably the Atacama Large Millimeter Array (ALMA) [67], [68], the Combined Array for Research in Millimeter-wave Astronomy (CARMA) ${ }^{2}$ [69], the Atacama Pathfinder Experiment (APEX) ${ }^{3}$ [70], South Pole Telescope (SPT) [71], and SOFIA [72], [73] all of which are either already operational or will become so within the next few years. As one might predict, the widespread development of submillimeter wave telescopes and instruments has led to a build-up of both scientists and technologists that in turn has resulted in a self sustaining interest spurring many new proposals, including applications for additional spaced-based systems. A few of these are highlighted in the section to follow.

\footnotetext{
${ }^{1}$ The most up to date and comprehensive information on Planck is available in the Planck Bluebook, see [66].

${ }^{2}$ CARMA just started operations and the latest information can be found at [69].

${ }^{3} \mathrm{APEX}$ is operational and observing programs can be submitted.
}

\section{PlanNED THz Space InStRUMENTS}

The success of Spitzer and Herschel as well as SWAS, MIRO, Odin, and MLS, have emboldened many Earth and space scientists to propose even more complex and technically challenging submillimeter-wave missions. A primary near term goal for spectral line astronomy is still the untapped information available from the strong oxygen line at 63 microns (4.76 $\mathrm{THz})$, the 112 micron HD line $(2.68 \mathrm{THz})$ as well as targeted mapping of the extremely abundant CII and NII lines at 1.9 and $1.46 \mathrm{THz}$, respectively. Many other species characterized by emission or absorption signatures at somewhat longer wavelengths, including water, lightweight oxygen and carbon compounds and deuterated molecules have been only very sparsely observed and play a strong role in stellar chemistry and evolution [74], [75]. Several middle explorer (MIDEX) targeted terahertz line survey mission proposals have already received significant attention within NASA over the years beginning withthe Submillimeter Moderate Mission $\left(\mathrm{SM}^{3}\right.$ [76]) in the mid 1990s and leading up to the recent Space Terahertz Observatory (STO [75]) proposal for the NASA Origins program. Submillimetron [77] is a small $(60 \mathrm{~cm})$ cooled $(4 \mathrm{~K})$ telescope with high sensitivity hot electron bolometer detector arrays out to $1.5 \mathrm{~mm}$ that the author was told just received the go-ahead for development and eventual deployment on the Russian section of the International Space Station. The major NASA follow-on to Spitzer and Herschel at the current time is an equally high tech reincarnation of LDR and has the acronym SAFIR (Single Aperture Far Infrared Observatory) [2], [78]. This $8-10 \mathrm{~m}$ diameter cooled $(5 \mathrm{~K})$ telescope with detectors from $1 \mathrm{~mm}$ to 20 microns is currently in the planning stages for the 2015 time frame [79]. Perhaps even more ambitious are a series of multidish interferometric telescope proposals under NASA (Submillimeter Probe of the Evolution of Cosmic Structure (SPECS) [2], [80] and Space Infrared Interferometric Telescope (SPIRIT) [2], [81]) and in the ESA community, Exploratory Submillimeter Space Radio-Interferometric Telescope (ESPRIT) [2], [82]. ESPRIT would field six $3.5 \mathrm{~m}$ diameter phase linked telescopes operating between 1.5 and 6 $\mathrm{THz}$ with 7-500 $\mathrm{m}$ baselines [83]. Space interferometry with its enhanced resolution capability would make a tremendous difference in the search for small cool bodies-like planets and planetary systems - a strong motivating driver for at least one close-to-home NASA study proposal $\mathrm{THz}$ Interferometer for Planets (TIP) [84].

On the Earth science side, terahertz spectral line receivers are confined to stratospheric measurements due to optical opacity in the troposphere (water and $\mathrm{O}_{2}$ absorption). As such there are fewer drivers for continued development of receivers at the higher $\mathrm{THz}$ frequencies. However, between 200 and $700 \mathrm{GHz}$ there are an enormous number of abundant and important emission lines that can be mapped and monitored for continued meteorological, pollution, climate change and atmospheric modeling information. A NASA follow-on program to Aura MLS that includes a comprehensive suite of climatology and meteorological measurement capabilities, Composition of the Atmosphere from Mid-Earth Orbit (CAMEO) [85] has recently been submitted for consideration as a near term atmospheric sounding mission. In Europe there is also very strong interest in better and more 
TABLE I

Past, Current, and Future Terahertz (and Precursor) Space Instruments Referenced in This PaPer

\begin{tabular}{|c|c|c|c|c|c|c|}
\hline $\begin{array}{l}\text { Mission or } \\
\text { Instrument }\end{array}$ & $\begin{array}{l}\text { Launch } \\
\text { Year }\end{array}$ & $\begin{array}{l}\text { Agency or } \\
\text { Country }\end{array}$ & Application & $\begin{array}{l}\text { Frequency } \\
\text { Coverage }\end{array}$ & Instrument Description & Status \\
\hline Mariner 2 & 1962 & NASA & Venus Flyby & $15.8 / 22.2 \mathrm{GHz}$ & Earliest $\mu$ wave observations & Completed \\
\hline $\begin{array}{l}\text { Cosmos } \\
243 \\
\text { Cosmos } \\
384\end{array}$ & $\begin{array}{l}1968 \\
1970\end{array}$ & $\begin{array}{l}\text { Soviet } \\
\text { Union }\end{array}$ & Earth & $3-37 \mathrm{GHz}$ & $\begin{array}{l}\text { Water vapor and temperature } \\
\text { sounding }\end{array}$ & Completed \\
\hline Nimbus 5 & 1972 & NOAA & Earth & $22-60 \mathrm{GHz}$ & $\begin{array}{l}\text { Water vapor, temperature } \\
\text { profiling, ice/snow }\end{array}$ & Completed \\
\hline $\begin{array}{l}\text { Cosmos } \\
669\end{array}$ & 1974 & $\begin{array}{l}\text { Soviet } \\
\text { Union }\end{array}$ & $\begin{array}{l}\text { Earth and } \\
\text { Astrophysics }\end{array}$ & $\begin{array}{l}\approx 300 \mathrm{GHz} \text { to } \\
<60 \mu \mathrm{m}\end{array}$ & $\begin{array}{l}\text { 1st Earth \& space IR } \\
\text { measurements using cooled } \\
\text { bolometers }\end{array}$ & Completed \\
\hline Salyut 6 & 1978 & $\begin{array}{l}\text { Soviet } \\
\text { Union }\end{array}$ & Astrophysics & $\begin{array}{l}\approx 200 \mathrm{GHz} \text { to } \\
<20 \mu \mathrm{m}\end{array}$ & $\begin{array}{l}\text { Space station telescope with } \\
\text { active } 4 \mathrm{~K} \text { cooler and } \\
\text { bolometric detectors }\end{array}$ & Completed \\
\hline IRAS & 1983 & NASA & Astrophysics & $8-120 \mu \mathrm{m}$ & $\begin{array}{l}\text { Helium cryogen \& cooled } \\
\text { beryllium antenna }\end{array}$ & Completed \\
\hline COBE & 1989 & NASA & Astrophysics & $\begin{array}{l}30 \mathrm{GHz}-3 \mathrm{THz} \\
\text { and } 1-240 \mu \mathrm{m}\end{array}$ & $\begin{array}{l}\text { Cosmic background and IR } \\
\text { mapper with high resolution } \\
\text { interferometer \& photometers }\end{array}$ & Completed \\
\hline $\begin{array}{l}\text { UARS- } \\
\text { MLS }\end{array}$ & 1991 & NASA & Earth & $63,183,205 \mathrm{GHz}$ & $\begin{array}{l}1^{\text {st }} \text { heterodyne receivers for } \\
\text { high resolution spectroscopy of } \\
\text { stratospheric constituents }\end{array}$ & Completed \\
\hline SSM/T-2 & 1991 & NOAA/DoD & Earth & $91,150,183 \mathrm{GHz}$ & Heterodyne for water vapor & Completed \\
\hline ISO & 1995 & ESA & Astrophysics & $2-240 \mu \mathrm{m}$ & $\begin{array}{l}\text { Superfluid He, cooled dish, } \\
\text { Fabry Perot, photometers and } \\
\text { photoconductive detectors }\end{array}$ & Completed \\
\hline SWAS & 1998 & NASA & Astrophysics & $490,550 \mathrm{GHz}$ & $\begin{array}{l}1^{\text {st }} \text { submillimeter heterodyne } \\
\text { instrument: water, } \mathrm{O}_{2} \text { and } \mathrm{CO}\end{array}$ & Completed \\
\hline Odin & 2001 & Sweden & $\begin{array}{l}\text { Astrophysics } \\
\text { \& Earth }\end{array}$ & $\begin{array}{l}118,490-500 \\
540-580 \mathrm{GHz}\end{array}$ & $\begin{array}{l}\text { Heterodyne space \& Earth } \\
\text { sounder with Sterling cooler }\end{array}$ & Operational \\
\hline WMAP & 2001 & NASA & Astrophysics & $90 \mathrm{GHz}$ & Cosmic background w/MMIC's & Operational \\
\hline Spitzer & 2003 & NASA & Astrophysics & $5-140 \mu \mathrm{m}$ & $\begin{array}{l}\text { Cooled telescope, multiband } \\
\text { photometers \& spectrograph }\end{array}$ & Operational \\
\hline MIRO & 2004 & ESA & Comet & $188,560 \mathrm{GHz}$ & $\begin{array}{l}1^{\text {st }} \text { submillimeter-wave } \\
\text { planetary mission. Heterodyne } \\
\mathrm{H}_{2} \mathrm{O}, \mathrm{CO}, \mathrm{NH}_{3}, \mathrm{CH}_{3} \mathrm{OH}\end{array}$ & $\begin{array}{l}\text { Operational } \\
\text { (not yet at } \\
\text { rendezvous) }\end{array}$ \\
\hline Aura MLS & 2004 & NASA & Earth & $\begin{array}{l}118,180,240 \\
640,2520 \mathrm{GHz}\end{array}$ & $\begin{array}{l}1^{\text {st }} \text { Terahertz heterodyne } \\
\text { mission: ozone } \& \text { climate } \\
\text { change }\end{array}$ & Operational \\
\hline AKARI & 2006 & JAXA & Astrophysics & $1-180 \mu \mathrm{m}$ & $\begin{array}{l}\text { Cryogen plus active cooler for } \\
6 \mathrm{~K} \text { telescope, all sky survey }\end{array}$ & Operational \\
\hline Herschel & 2008 & ESA & Astrophysics & $\begin{array}{l}480-1900 \mathrm{GHz} \\
\text { plus } 60-200 \mu \mathrm{m}\end{array}$ & $\begin{array}{l}\text { Heterodyne and direct detection } \\
\text { receivers, cooled telescope, } \\
\text { multiple instruments }\end{array}$ & $\begin{array}{l}\text { In } \\
\text { Construction }\end{array}$ \\
\hline Planck & 2008 & ESA & Astrophysics & $\begin{array}{l}30-70 \mathrm{GHz} \\
100-850 \mathrm{GHz}\end{array}$ & $\begin{array}{l}\text { Cosmic background with high } \\
\text { and low frequency channels }\end{array}$ & $\begin{array}{l}\text { In } \\
\text { Construction }\end{array}$ \\
\hline SMILES & 2008 & JAXA & Earth & $625-650 \mathrm{GHz}$ & $\begin{array}{l}1^{\text {st }} \text { superconducting heterodyne } \\
\text { system for Earth observations }\end{array}$ & $\begin{array}{l}\text { Completed, } \\
\text { awaiting } \\
\text { launch }\end{array}$ \\
\hline Vesper & $2012+$ & NASA & Venus & $460-560 \mathrm{GHz}$ & $\begin{array}{l}\text { High resolution spectroscopy } \\
\text { for clouds and winds }\end{array}$ & $\begin{array}{l}\text { Approved } \\
\text { Concept }\end{array}$ \\
\hline $\begin{array}{l}\text { Submilli- } \\
\text { metron }\end{array}$ & $2015 ?$ & Russia & Astrophysics & $150-1500 \mathrm{GHz}$ & $\begin{array}{l}\text { Int. Space Station project, } \\
\text { cooled dish, He bolometers }\end{array}$ & $\begin{array}{l}\text { Approved } \\
\text { Concept }\end{array}$ \\
\hline $\begin{array}{l}\text { Future } \\
\text { Astronomy }\end{array}$ & $>2015$ & NASA/ESA & Astrophysics & mm-to-IR & $\begin{array}{l}\text { SAFIR, SPIRIT, SPECS, } \\
\text { ESPRIT, STO, TIP... }\end{array}$ & $\begin{array}{l}\text { Proposals } \\
\text { Concepts }\end{array}$ \\
\hline $\begin{array}{l}\text { Future } \\
\text { Earth }\end{array}$ & $>2012$ & NASA/ESA & Earth & mm-submm & $\begin{array}{l}\text { CAMEO, ACECHEM, } \\
\text { meteorological } \\
\text { interferometers... } \\
\end{array}$ & $\begin{array}{l}\text { Proposal } \\
\text { Concepts }\end{array}$ \\
\hline $\begin{array}{l}\text { Future } \\
\text { Planets }\end{array}$ & $>2015$ & NASA/ESA & Planets & mm-submm & Mars, Titan, Europa ... & $\begin{array}{l}\text { Proposal } \\
\text { Concepts }\end{array}$ \\
\hline
\end{tabular}

timely meteorological forecasting, and at least one very exciting large aperture interferometric array concept is being seriously proposed for continuous Earth monitoring from the Lagrange points [86]. Almost every country has a strong interest in Earth 
science as related to weather forecasting, resource distribution or pollution monitoring and there are many more proposals that have been put forward for instruments using millimeter and submillimeter wave spectral signatures to look at water, oxygen and dozens of other chemical tracers. The reader is referred to the websites of the major space agencies throughout the world, including China (CSNA) [87] and India (ISRO) [88], as well as the long running conference series the International Symposium on Space Terahertz Technology (ISSTT) ${ }^{4}$ and International Symposium on Infrared and Millimeter Waves and Terahertz Electronics (IRMMW-THz) [89] and the many targeted conferences by SPIE, especially the alternate year series on Astronomical Telescopes and Instrumentation [90] to keep abreast of developments in this arena.

Finally, there are almost as many proposals submitted or pending within NASA for using submillimeter wave spectral line sensors as there are planets to visit in the solar system, since orbital measurements provide global coverage of atmospheric composition, dynamics and radiance. In particular, on Mars it is possible to limb scan all the way down to within a few kilometers of the surface between 500 and $700 \mathrm{GHz}$ and possibly detect emission spectra from subsurface volcanic plumes as well as species indicative of life [4]. A mission to study the cloud composition and temperature distribution in the atmosphere of Venus using $450-560 \mathrm{GHz}$ spectrometers is on the short list of this year's Discovery proposal selection process [91]. Proposals for Titan and Europa as well as a Mars lander and other orbiters have also been submitted over the years. The author predicts that at least one of these planetary proposals will be funded in this decade as scientific interest in this wavelength region and technological maturity continue to converge.

\section{SUMMARY}

The author has attempted to survey the past, present and future submillimeter-wave and terahertz space instruments (see Table I) and some of their science applications. Clearly the number of programs and the number of scientists and technologists is growing at an impressive rate. As the technology improves it is likely that even more terahertz space instruments will be proposed, as their science justification goes back to the earliest days of radio astronomy and meteorological observations from space. Many exciting proposals have already made their way into space agencies in the U.S., Europe and Japan. China and India have now entered the space race with a passion. Despite the belt tightening being felt by many scientists, the investments already made in Herschel and Planck, ALMA APEX, CARMA, SPT, ODIN, SWAS, MIRO, MLS SOFIA, etc., clearly define this era as the golden age for terahertz science and instruments.

\section{ACKNOWLEDGMENT}

Hopefully, the author has not offended too many people or institutions by inadvertently neglecting to mention their programs or their proposals. He expresses his deep regret and apologizes in advance for these omissions. For the information that

\footnotetext{
${ }^{4}$ Individual conference abstracts can usually be found at websites containing ISSTT or STT in the name. Full conference digest archives will soon be available for the 17 individual symposia to date.
}

is contained within the paper, the author would like to make the following acknowledgements. First to all the past and current members of the JPL Submillimeter Wave Advanced Technology team for their many contributions to the field of space $\mathrm{THz}$ technology and with whom the author has been associated for the past 12 years. He would also like to acknowledge the following individuals who provided information or references used in this review article: Dr. P. Goldsmith (JPL), Dr. A. I. Nosich (Kharkov, Ukraine) and Prof. Y. Kuleshov of the P.N. Lebedev Physics Institute, Russia, for the information on the submillimeter telescope on Salyut 6. Mr. I. Galin (NGC, Azusa, CA) for references on DMSP. This work was carried out at the Jet Propulsion Laboratory and the California Institute of Technology under grants from the National Institutes of Health, and the National Aeronautics and Space Administration.

\section{REFERENCES}

[1] T. G. Phillips and J. C. Keene, "Submillimeter astronomy," Proc. IEEE, vol. 80, no. 11, pp. 1662-1678, Nov. 1992.

[2] "From Spitzer to Herschel and Beyond: The Future of Far-Infrared Space Astrophysics," in Hosted by the JPL Center for Long Wavelength Astrophysics (CLWA) and the Caltech Infrared Processing and Analysis Center (IPAC). Pasadena, CA, Jun. 7-10, 2004 [Online]. Available: http://safir.jpl.nasa.gov/BeyondSpitzerConf/confProceedings.shtml

[3] J. W. Waters, "Submillimeter-wavelength heterodyne spectroscopy and remote sensing of the upper atmosphere," Proc. IEEE, vol. 80, no. 11, pp. 1679-1701, Nov. 1992.

[4] S. Matousek, "Mars scout 2007-A current status," in IEEE Aerospace Conf., 2003, vol. 2, pp. 739-743.

[5] M. A. Janssen, Atmospheric Remote Sensing by Microwave Radiometry. New York: Wiley, 1993.

[6] E. G. Njoku, "Passive microwave remote sensing of the earth from space,” Proc. IEEE, vol. 70, no. 7, pp. 728-750, Jul. 1982.

[7] D. Staelin, "Passive microwave techniques for geophysical sensing of the earth from satellites," IEEE Trans. Antennas Propag., vol. 29, no. 4, pp. 683-687, Jul. 1981.

[8] J. W. Waters et al., "The UARS and EOS microwave limb sounder (MLS) experiments," J. Atmospheric Sci., vol. 56, no. 2, pp. 194-218, Feb. 1999.

[9] J. W. Waters, L. Froidevaux, W. G. Read, G. L. Manney, L. S. Elson, D. A. Flower, R. F. Jarnot, and R. S. Harwood, "Stratospheric ClO and ozone from the Microwave Limb Sounder on the upper atmosphere research satellite," Nature, vol. 362, pp. 597-602, 1993.

[10] I. Galin, D. H. Brest, and G. R. Martner, "The DMSP SSMT/2 microwave water-vapor profiler," in SPIE OE/Aerospace and Remote Sensing Int. Symp., Orlando, FL, 1993.

[11] I. Galin, "The receiver subsystem for the AMSU-B water vapor profiler,' in SPIE Int. Conf. on MM-Waves and Sub-MM-Waves, San-Diego, CA, 1994.

[12] A. E. Salomonovich, "Extra-atmospheric submillimeter astronomy," Soviet Physics Uspekhi, vol. 12, no. 6, pp. 731-742, May-Jun. 1970.

[13] L. Z. Dul'kin, A. D. Karelin, R. P. Nikolaev, and G. R. Pekki, "Exoatmospheric optoelectronic packages for the study of space: Development experience and basic results," J. Opt. Technol., vol. 67, no. 5, pp. 485-492, May 2000.

[14] A. E. Salomonovich et al., "A submillimeter telescope for the orbital piloted station Salyut-6," Telecomm. Radio Eng., vol. 34, pt. 2, pp. 82-88, May 1979, (translation of Radiotekhnika, vol. 34, May 1979, pp. 33-40).

[15] G. W. Chantry, in Submillimetre waves and their applications: Proc. 3rd Int. Conf., Guildford, U.K., Dec. 1978, vol. 18.

[16] E. J. Blum, "Radioastronomy at millimeter wavelengths," in Advances in Electronics and Electronic Physics. New York: Academic, 1981, vol. 56, pp. 97-162.

[17] J. C. Wiltse, "History of millimeter and submillimeter waves," IEEE Trans. Microw. Theory Tech., vol. 32, no. 9, pp. 1118-1127, Sep. 1984.

[18] R. W. Wilson, K. B. Jefferts, and A. A. Penzias, "Carbon monoxide in the Orion nebula," Astrophys. J., vol. 161, no. 1, pt. 2, pp. L43-4, Jul. 1970.

[19] R. K. Kakar, J. W. Waters, and W. J. Wilson, "Venus: Microwave detection of CO in the venus stratosphere," Science, vol. 191, pp. 379-380, Jan. 30, 1976. 
[20] B. T. Soifer and J. L. Pipher, "Instrumentation for Infrared Astronomy," Annu. Rev. Astronomy Astrophys., vol. 16, pp. 335-369, 1978.

[21] G. V. Schultz, E. J. Durwen, H. P. Roser, W. A. Sherwood, and R. Wallenbach, "Detection of the $\mathrm{CO}(\mathrm{J}=7-6)$ rotational transition at lambda $=0.37$ millimeters toward Orion," Astrophys. J., vol. 291, no. 2, pt. 2, pp. 59-61, Apr. 15, 1985, Letters to the Editor.

[22] G. J. Stacey and N. T. Kurtz et al., "The mass of hot, shocked CO in Orion: First observations of the $\mathrm{J}=17$ to $\mathrm{J}=16$ transition at 153 microns," Astrophys. J., vol. 257, no. 1, pt. 2, pp. L37-40, Jun. 1, 1982, Letters to the Editor.

[23] B. Carli, F. Mencaraglia, and A. Bonetti, "Submillimeter high resolution FT spectrometer for atmospheric studies," Appl. Opt., vol. 23, no. 15, pp. 2594-2603, Aug. 1, 1984.

[24] H. M. Pickett, R. L. Poynter, E. A. Cohen, M. L. Delitsky, J. C. Pearson, and H. S. P. Muller, "Submillimeter, millimeter, and microwave spectral line catalog," J. Quant. Spectroscopy Radiative Transfer, vol. 60, pp. 883-890, 1998.

[25] G. Neugebauer et al., "The infrared astronomical satellite (IRAS) mission," Astrophys. J., vol. 278, pt. 2, pp. L1-L6, Mar. 1, 1984, Letters to the Editor.

[26] G. Neugebauer, C. A. Beichman, and B. T. Soifer et al., "Early results from the infrared astronomical satellite," Science, vol. 224, no. 4644, pp. 4-21, Apr. 6, 1984.

[27] M. F. Kessler, J. A. Steinz, and M. E. Anderegg et al., "The infrared space observatory (ISO) mission," Astron. Astrophys., vol. 315, pp. L27-L31, 1996.

[28] M. Kessler, J. Clavel, and J. Faelker, "Looking back at ISO operations," ESA Outreach Bulletin 95 [Online]. Available: http://www.iso.esac. esa.int/outreach/bck_grnd/bull/95.pdf

[29] P. E. Clegg et al., "The ISO long-wavelength spectrometer," Astron. Astrophys., vol. 315, pp. L38-L42, 1996.

[30] M. F. Kessler, , D. Lemke, M. Stickel, and K. Wilke, Eds., "The ISO Mission and Its Surveys," in Lecture Notes in Physics. Berlin, Germany: Springer Verlag, 2000, vol. 548, pp. 3-10.

[31] N. W. Boggess, J. C. Mather, and R. Weiss et al., "The COBE mission-Its design and performance two years after launch," Astrophys. J., vol. 397, no. 2, pt. 1, pp. 420-429, Oct. 1992.

[32] [Online]. Available: http://aether.lbl.gov/www/projects/cobe/

[33] [Online].Available:http://map.gsfc.nasa.gov/m_mm/ob_techsummary. html

[34] [Online]. Available: http://www.spitzer.caltech.edu/

[35] M. W. Werner et al., "The spitzer space telescope mission," Special Issue Astrophys. J. Supplement Series, vol. 154, Sep. 2004.

[36] F. Lahuis, E. F. Van Dishoeck, A. C. A. Boogert, K. M. Pontoppidan, G. A. Blake, C. P. Dullemond, N. J. Evans, M. R. Hogerheijde, J. K. Jørgensen, J. E. Kessler-Silacci, and C. Knez, "Hot organic molecules toward a young low-mass star: A look at inner disk chemistry," Astrophys. J. Lett., vol. 636, pp. L145-L148, Jan. 10, 2006.

[37] G. Blake, Calif. Inst. of Tech. [Online]. Available: http://www.spitzer. caltech.edu/Media/releases/ssc2005-26/release.shtml

[38] T. Nakagawa, ASTRO-F Survey as Input Catalogues for FIRST [Online]. Available: http://www.ir.isas.jaxa.jp/ASTRO-F/detail/general2/index.html from:

[39] G. Melnick et al., "The submillimeter wave astronomy satellite: Science objectives and instrument description," Astrophys. J. Lett., vol. 539, no. 2, pt. 2, pp. L77-L85, Aug. 20, 2000.

[40] F. Bensch, G. Melnick, D. Neufeld, M. Harwit, R. Snell, and B. M. Patten, "SWAS observations of comet 9P/Tempel 1 and deep impact," in Proc. Int. Astronomical Union Symp. S231, Aug. 2005, vol. 1, pp. 489-490.

[41] [Online]. Available: http://www.ssc.se/default.asp?groupid=20052 1610221402

[42] "First Science with the Odin Satellite," in Special Issue of Astron. Astrophys. vol. 402/3, pp. L21-L81, 805-1158, May 2003.

[43] [Online]. Available: http://directory.eoportal.org/pres_Odin.html

[44] A. Hjalmarson et al., "Highlights from the first year of Odin observations," Astron. Astrophys., vol. 402, no. 3, pp. L39-L46, May 2003.

[45] [Online]. Available: http://www.ssc.se/default.asp?groupid=2005216 10221402\&pageid $=2006221145129730$

[46] J. W. Waters et al., "The UARS and EOS microwave limb sounder (MLS) experiments," J. Atmos. Sci., vol. 56, no. 2, pp. 194-218, Feb. 1999.

[47] M. C. Gaidis, H. M. Pickett, C. D. Smith, R. P. Smith, S. C. Martin, and P. H. Siegel, "A $2.5 \mathrm{THz}$ receiver front-end for spaceborne applications," IEEE Trans. Microwave Theory Tech., vol. MTT-48, no. 4, pp. 733-739, Apr. 2000.
[48] S. Gulkis, M. Frerking, G. Beaudin, P. Hartogh, M. Janssen, C. Kahn, T. Koch, and Y. Salinas, "Microwave instrument for the Rosetta orbiter (MIRO)," in ESA-SP 1165. Noordwijk: The Netherlands, 2002, (special publication).

[49] [Online]. Available: http://sci.esa.int/science-e/www/object/index. $\mathrm{cfm}$ ? fobjectid $=35061 \&$ fbodylongid $=1641$

[50] J. Inatani et al., "Submillimeter limb-emission sounder JEM/SMILES aboard the space station," in Proc. SPIE, Nara, Japan, Oct. 9-12, 2000, vol. 4152, Remote Sensing of the Atmosphere, Environment and Space, session 4152-36.

[51] [Online]. Available: http://smiles.tksc.jaxa.jp/introduction/pamphlete. html

[52] P. Swanson, "Large space antenna (LSA) configuration study for radio astronomy application," JPL Internal Rep. 710-5, Mar. 1977.

[53] P. Swanson, J. B. Breckinridge, A. Diner, R. E. Freeland, W. R. Irace, P. M. McElroy, A. B. Meinel, and F. A. Tolivar, "A system concept for a moderate cost large deployable reflector (LDR)," Opt. Eng., vol. 25, no. 9, Sep. 1986.

[54] P. Swanson, "The large deployable reflector (LDR): Plans and progress," presented at the Radio Astronomy From Space Workshop Held at the National Radio Astronomy Observatory, Green Bank, WV, 30 Sep. -2 Oct. 1986.

[55] G. Winnewisser, "Submillimetre wave spectroscopy in astronomy related to the ESA-project FIRST (far infrared submillimetre space telescope)," Proc. SPIE, vol. 598, pp. 2-7, 1986.

[56] G. Pilbratt, "The ESA FIRST cornerstone mission," Proc. SPIE UV, Optical, and IR Space Telescopes and Instruments, vol. 4013, pp. 142-151, 2000.

[57] [Online]. Available: http://sci.esa.int/science-e/www/area/index.cfm? fareaid $=16$

[58] E. Sein, Y. Toulemont, F. Safa, M. Duran, P. Deny, D. Chambure, T. Passvogel, and G. Pilbratt, "A $\Phi$ 3.5 M SiC telescope for herschel mission," Proc. SPIE, IR Space Telescopes and Instruments, vol. 4850, pp. 606-618, 2003.

[59] M. W. de Graauw et al., "The heterodyne instrument for first (HIFI): Preliminary design," Proc. SPIE, vol. 3357, pp. 336-347, Jul. 1998.

[60] N. D. Whyborn, The HIFI Heterodyne Instrument for FIRST: Capabilities and Performance. France: ESA Special Publication, 1997, vol. SP-401, pp. 19-24 [Online]. Available: http://www.rssd.esa.int/Herschel/Publ/1997/sp401-het.pdf

[61] T. de Graauw et al., "The herschel-heterodyne instrument for the far infrared (HIFI)," in Proc. SPIE, Conf. on Astronomical Telescopes and Instrumentation, Orlando, FL, May 24-31, 2006.

[62] M. Griffin, B. Swinyard, and L. Vigroux, The SPIRE Instrument for Herschel. France: ESA Special Publication, 2001, vol. 460, pp. 37-44 [Online]. Available: http://www.rssd.esa.int/SA-general/ Projects/Herschel/Publ/2001/sp460_toledo_spire.pdf

[63] A. Turner, J. Bock, J. Beeman, J. Glenn, P. Hargrave, V. Hristov, H. T. Nguyen, F. Rahman, S. Sethuraman, and A. Woodcraft, "Silicon nitride micromesh bolometer array for submillimeter astrophysics," Appl. Opt., vol. 40, no. 28, pp. 4921-4932, Oct. 2001.

[64] A. Poglitsch et al., "The photodetector array camera \& spectrometer (PACS) for the herschel space observatory," Proc. SPIE, vol. 6265, Jul. 2006, Space Telescopes and Instrumentation.

[65] J. A. Tauber, "The Planck mission," Advances in Space Res., vol. 34, no. 3, pp. 491-496, 2004.

[66] [Online]. Available: http://www.rssd.esa.int/SA/PLANCK/docs/Bluebook-ESA-SCI(2005)1.pdf

[67] [Online]. Available: http://www.alma.info/

[68] "Recent ALMA Papers," Proc. XXVIth General Assembly Meeting of the International Astronomical Union. Prague, Czechoslovakia, Aug. $10-25,2006$.

[69] [Online]. Available: www.mmarray.org/intro.html

[70] [Online]. Available: http://www.apex-telescope.org/

[71] J. E. Ruhl et al., "The south pole telescope," Proc. SPIE, vol. 5498, pp. 11-29, 2004

[72] E. F. Erickson, "SOFIA: Stratospheric observatory for infrared astronomy," Space Sci. Rev., vol. 61, no. 1-2, pp. 61-68, 1992.

[73] E. E. Becklin, "Stratospheric observatory for infrared astronomy (SOFIA)," Advances Space Res., vol. 36, no. 6, pp. 1087-1090, 2005.

[74] T. G. Phillips and C. Vastel, "Future MM/Submm instrumentation and science opportunities: Examples of deuterated molecules," presented at the Chemistry as Diagnostic of Star Formation Conf., Waterloo, ON, Canada, Aug. 21-23, 2002.

[75] C. Walker et al., "The space terahertz observatory (STO: A 10-metercalss far-infrared telescope for origins research," NASA Origins Proposal 2005 . 
[76] T. G. Phillips, Y. Kondo, Ed., "A submillimeter mission for the 1990s-SMMM," IAU Colloq. 123, Observatories in Earth Orbit and Beyond ASSL, vol. 166, pp. 231-249, 1990.

[77] V. D. Gromov et al., "Submillimeter telescope for the Russian segment of the ISS: Submillimetron project," in Proc. Promise of FIRST Symp., Toledo, Spain, 2001, vol. ESA SP-460.

[78] D. Lester, D. Benford, H. Yorke, C. M. Bradford, K. Parrish, and H. Stahl, "Science promise and conceptual mission design for SAFIR: The single aperture far-infrared observatory," Proc. SPIE, vol. 6265, Jul. 2006, paper 6251X.

[79] [Online]. Available: http://safir.jpl.nasa.gov/

[80] J. C. Mather et al., The Submillimeter Frontier: A Space Science Imperative. Aug. 11, 2006 [Online]. Available: http://www.citebase.org/fulltext?format=application\%2Fpdf\&identifier=oai\%3AarXiv.org\%3 Aastro-ph\%2F9812454

[81] [Online]. Available: http://www.nasa.gov/centers/goddard/news/topstory/2004/0915spirit_prt.htm

[82] W. Wild et al., "ESPRIT: A space interferometer concept for the far-infrared," Proc. SPIE, Space Telescopes and Instrumentation, vol. 6265, Jul. 2006.

[83] T. de Graauw, "FIR space heterodyne interferometer mission (ESPRIT)," in 35th COSPAR Scientific Assembly, Paris, France, Jul. $18-25,2004$, p. 4564.

[84] G. Chattopadhyay, J. Ward, I. Mehdi, P. H. Siegel, P. Goldsmith, and A. Readhead, "TIP: A THz interferometer for planets-a concept study," presented at the 17th Int. Symp. on Space THz Technology, ISTT2006, Paris, France, May 10-12, 2006.

[85] J. W. Waters et al., "Composition of the atmosphere from mid earth orbit (CAMEO)," Proposal to U.S. National Research Council Decadal Survey on Earth Science and Applications from Space, May 2005.

[86] U. Klein, "Future satellite earth observation requirements and technology in millimetre and sub-millimetre wavelength region," in 17th Int. Symp. on Space THz Technology, Paris, France, May 10-12, 2006, paper WE plenary\#3.

[87] Chinese National Space Administration, [Online]. Available: http://202.106.142.5/main_e.asp

[88] Indian Space Research Organization, [Online]. Available: http://www. isro.org/

[89] Int. Conf. on Infrared and Millimeter Waves and Terahertz Electronics [Online]. Available: www.irmmw-thz.org

[90] SPIE Conf. on Astronomical Telescopes and Instrumentation, [Online]. Available: http://spie.org/app/conferences/index.cfm 1998, 2000, 2002, 2004, 2006

[91] G. Chin et al., "Vesper, an infrared and submillimeter wave limb scanning satellite for Venus containing SLS-submillimeter line spectrometer (450-560 GHz)," NASA Discovery Proposal Apr. 2006.

[92] M. Allen et al., "Marvel: Mars volcanic emission life scout," proposal for NASA Scout Mission opportunity, Jul. 2006.

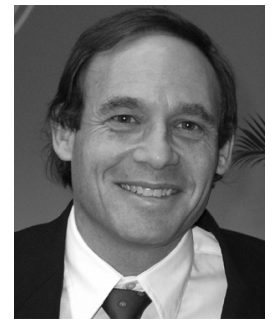

Peter H. Siegel (F'93) received the BA degree in astronomy from Colgate University, Hamilton, NY, in 1976, and the M.S. degree in physics and the Ph.D. degree in electrical engineering from Columbia University, New York, in 1978, and 1983, respectively.

$\mathrm{He}$ has been involved in the analysis and development of millimeter- and submillimeter-wave sensors for more than 30 years. From 1975 to 1983 , he worked on millimeter wave receiver development at the NASA Goddard Institute for Space Studies in New York. Following a National Research Council Postdoctoral Fellowship in 1984, he spent three years at the Central Development Laboratory of the National Radio Astronomy Observatory in Charlottesville, VA. He joined the NASA Jet Propulsion Laboratory, California Institute of Technology, Pasadena, in 1987 where he began working on terahertz superconductor and semiconductor receivers for advanced space instrument concepts. In 1993, he formed the JPL Submillimeter Wave Advanced Technology (SWAT) team, a group of some 25 engineers and scientists working on the development of submillimeter-wave technology for NASA's near and long term space astrophysics, Earth remote sensing, and planetary mission applications. The team has so far delivered submillimeter wave hardware to four space flight instruments, and he has been principal investigator or co-investigator on more than 65 competitively won programs in the $\mathrm{THz}$ area. In 2002, he joined the staff at the California Institute of Technology as a Senior Scientist at the Beckman Institute, Division of Biology, where he is currently working on a variety of $\mathrm{THz}$ biomedical applications. He maintains a joint appointment as the Technical Group Supervisor for SWAT at JPL, where he continues to propose and work on space applications of THz technology.

Dr. Siegel is a member of the American Association for the Advancement of Science (AAAS). He received the first U.S. National Institute of Health grant to work on terahertz applications in the health sciences, in 2002. He is the chair of IEEE MTT Committee 4-Terahertz Techniques and Applications, ViceChair of the International Organizing Committee for the Infrared and Millimeter Waves and Terahertz Electronics Conference Series. He has served as an IEEE Distinguished Microwave Lecturer and has been an Organizer and Chair of seven special sessions on terahertz techniques and applications at the annual IEEE International Microwave Symposium. Finally, he is scheduled to chair the 33rd IRMMW-THz conference at Caltech in Pasadena, CA, in 2008. 